\title{
ABORDAGEM DA SAÚDE ÚNICA NA OCORRÊNCIA DE ENTEROPARASITAS EM HUMANOS DE ÁREA URBANA NO NORTE DO PARANÁ
}

\author{
Aline do Nascimento Benitez ${ }^{1}$ \\ Marcelle Mareze ${ }^{2}$ \\ Ana Carolina Miura ${ }^{3}$ \\ Deborah Thais Silva Cepelo Brunieri ${ }^{4}$ \\ Fernanda Pinto Ferreira ${ }^{5}$ \\ Regina Mitsuka-Breganó ${ }^{6}$ \\ Italmar Teodorico Navarro ${ }^{7}$
}

BENITEZ, A. do N.; MAREZE, M.; MIURA, A. C.; BRUNIERI, D. T. S. C.; FERREIRA, F. P.; MITSUKA-BREGANÓ, R.; NAVARRO, I. T. Abordagem da Saúde Única na ocorrência de enteroparasitas em humanos de área urbana no Norte do Paraná. Arq. Ciênc. Vet. Zool. UNIPAR, Umuarama, v. 19, n. 4, p. 203-208, out./dez. 2016.

RESUMO: As enteroparasitoses causadas por helmintos e protozoários infectam humanos e animais no mundo todo, e sua prevalência está relacionada à falta de hábitos de higiene pessoal em consequência do seu ciclo oral-fecal. No Brasil, como não são doenças de notificação obrigatória, é possível que sua prevalência esteja subnotificada. Em Londrina, não existem dados sobre a prevalência dessas parasitoses na população urbana adulta, que ofereçam suporte para delinear programas de saúde preventiva na comunidade. Foram coletadas amostras de fezes de 187 humanos residentes na área urbana de Londrina para pesquisa de enteroparasitas. Dados epidemiológicos foram coletados por meio de um questionário epidemiológico aplicado a todos os participantes. As variáveis associadas ao risco foram analisadas conforme foram relatadas pelos indivíduos parasitados. Foram discutidos: gênero, idade, renda familiar mensal, lavagem das mãos antes da alimentação e após utilizar o banheiro, água de consumo, ocorrência de diarreia nos últimos sete dias, abastecimento de água e rede de esgoto. Foram detectados ovos ou cistos de parasitas em 8/187 (4,27\%) amostras investigadas. A ocorrência do protozoário Giardia spp. foi verificada em 7/187 (3,74\%) amostras e o helminto Enterobius vermiculares em 1/187 (0,53\%). Devido ao ciclo destes parasitas envolver a saúde humana, animal e do ambiente, é sugerido que a ocorrência das enteroparasitoses reduza por meio da abordagem de Saúde Única na área urbana de Londrina, PR.

PALAVRAS-CHAVE: Enteroparasitoses. Giardia spp. Saúde Pública. Zoonoses.

\section{SINGLE HEALTH APPROACH IN THE OCCURRENCE OF ENTEROPARASITES IN HUMANS IN THE URBAN AREA IN NORTHERN PARANÁ}

\begin{abstract}
Intestinal parasitic infections are caused by helminthes and protozoa infecting humans and animals worldwide. Its prevalence is related to lack of personal hygiene habits due to its oral-fecal cycle. In Brazil, they do not require compulsory notification and, therefore, it is possible that the prevalence is underreported. In Londrina, there are no data on the prevalence of these parasites in the adult urban population to support the designing of preventive health programs in the community. Stool samples were collected from 187 human residents in the urban area of Londrina in order to research for enteroparasites. Parasite eggs or cysts were detected in 8/187 (4.27\%) samples investigated. The occurrence of Giardia spp. was seen in 7/187 (3.74\%) samples, while Enterobius vermicularis was observed in 1/187 (0.53\%) sample. Epidemiological data were collected using an epidemiological questionnaire applied to all participants. The variables associated with the risk were analyzed and reported by infected individuals, such as: gender, age, family income, washing hands before eating and after using the bathroom, water consumption, occurrence of diarrhea in the past seven days, water supply and sewage system. Since the parasite cycle involves human, animal and environmental health, it is suggested that the occurrence of intestinal parasites be reduce through the Single Health approach in the urban area of Londrina, PR.
\end{abstract}

KEYWORDS: Enteroparasitosis. Giardia spp. Public Health. Zoonosis.

DOI: https://doi.org/10.25110/arqvet.v19i4.2016.6097

'Doutorado no Programa de Pós-Graduação em Ciência Animal, Universidade Estadual de Londrina, PR, 86057-970, Brasil. Email: benitez.alinenascimento@gmail.com

${ }^{2}$ Mestrado no Programa de Pós-Graduação em Ciência Animal, Universidade Estadual de Londrina, PR, 86057-970, Brasil.

Email: marcelle mareze@hotmail.com

${ }^{3}$ Doutorado no Programa de Pós-Graduação em Ciência Animal, Universidade Estadual de Londrina, PR, 86057-970, Brasil. Email: carolmiuravet@gmail.com

${ }^{4}$ Espacialização no Programa de Residência em Saúde Pública e Zoonoses, Universidade Estadual de Londrina, PR, 86057-970, Brasil.

Email: deborah.brunieri@gmail.com

${ }^{5}$ Mestrado no Programa de Pós-Graduação em Ciência Animal, Universidade Estadual de Londrina, PR, 86057-970, Brasil.

Email: nandaferreiravet@gmail.com

${ }^{6}$ Docente em Parasitologia Veterinária, Departamento de Medicina Veterinária Preventiva, Universidade Estadual de Londrina, PR, 86057-970, Brasil. Email: rbregano@gmail.com

${ }^{7}$ Docente em Zoonoses e Saúde Pública, Departamento de Medicina Veterinária Preventiva, Universidade Estadual de Londrina, PR, 86057-970, Brasil. Email: italmar@uel.br 


\section{ENFOQUE DE LA SALUD ÚNICA EN OCURRENCIA DE ENTEROPARÁSITOS EN HUMANOS DE LA ZONA URBANA EN EL NORTE DE PARANÁ}

RESUMEN: Las enteroparasitosis causadas por helmintos y protozoarios infectan humanos y animales en todo el mundo, y su prevalencia está relacionada con la falta de hábitos de higiene personal, como resultado de su ciclo fecal-oral. En Brasil, no son enfermedades de declaración obligatoria, es posible que su prevalencia no se denuncie. En Londrina, no hay datos sobre la prevalencia de esos parásitos en la población adulta urbana, que apoyen el diseño de programas preventivos de salud en la comunidad. Se recogieron muestras de heces de 187 residentes humanos en el área urbana de Londrina para investigación de enteroparásitos. Datos epidemiológicos se han recogido mediante un cuestionario epidemiológico administrado a todos los participantes. Las variables asociadas al riesgo se analizaron conforme relatado por los individuos infectados. Se discutieron: género, edad, renta familiar mensual, lavarse las manos antes de comer y después de ir al baño, agua de consumo, la aparición de diarrea en los últimos siete días, el suministro de agua y alcantarillado. Se ha detectado huevos o quistes de parásitos en 8/187 (4,27\%) muestras investigadas. La aparición del protozoário Giardia spp. se observó en 7/187 (3,74\%) muestras y el helminto Enterobius vermicularis en 1/187 (0,53\%). Debido al ciclo de estos parásitos involucrar la salud humana, animal y el medio ambiente, se sugiere que la aparición de enteroparasitosis se reduzca a través del enfoque de Salud Única en la zona urbana de Londrina, PR.

PALABRAS CLAVE: Enteroparasitosis. Giardia spp. Salud Pública. Zoonosis.

\section{Introdução}

Os enteroparasitas de alta incidência, como os helmintos e os protozoários, afetam a saúde humana e causam preocupação em saúde pública (NEVES et al., 2011). O protozoário Giardia spp., lidera entre as gastroenterites infecciosas negligenciadas em todo o mundo (SAVIOLI, et al., 2006). Estima-se que 400 milhões de pessoas no mundo estejam infectadas por este parasita (BORGES, et al., 2011). Mamíferos, anfíbios e aves podem ser acometidos por várias espécies do gênero Giardia spp. (WHO, 1996).

Com relação às helmintoses, mais de 1,5 bilhões pessoas ou $24 \%$ da população mundial estão infectadas, com uma maior distribuição entre as áreas tropicais e subtropicais (WHO, 2016). Em municípios com baixo índice de desenvolvimento humano (IDH), estima-se valores de prevalência de geohelmintos entre 2 a 36\% (BRASIL, 2012). Nos países em desenvolvimento, esta situação se agrava devido ao crescimento desordenado das cidades, que resulta em problemas de planejamento estrutural e desigualdade social (PRADO et al., 2001).

As manifestações clínicas variam conforme a carga parasitária, podendo provocar diarreia, má absorção intestinal, obstrução intestinal, colite, anemia e desnutrição, resultando em prejuízos ao indivíduo e à economia, especialmente associada à força de trabalho dos manipuladores de alimentos (COLLI et al., 2014). Crianças são mais suscetíveis à infecção e podem desenvolver deficiência física, nutricional e cognitiva quando infectadas (OMS, 2015).

Entre os principais fatores de risco associados às infecções parasitárias, estão falta de saneamento básico, deficientes características sócio-econômico-educacionais, aglomeração de pessoas, consumo de água não tratada e a contaminação dos alimentos (LOPES et al., 2006; PAES et al., 1999). Além da influência da localidade e população alvo estudadas, a prevalência das parasitoses varia conforme os métodos de detecção empregados (GOMES et al., 2011).

O presente estudo teve como objetivo determinar a ocorrência de enteroparasitas em 187 indivíduos na população adulta da cidade de Londrina, Estado do Paraná e verificar a possibilidade de aplicação dos conceitos de Saúde Única a partir dos resultados.

\section{Material e Métodos}

Este trabalho foi realizado após aprovação do Comitê de Ética em Pesquisa da Universidade Estadual de Londrina (CEP/UEL), registrado sob o $\mathrm{n}^{\circ} 1.025 .861 / 2014$.

$\mathrm{O}$ estudo foi realizado na área urbana do município de Londrina, PR, no período de junho a setembro de 2015. A cidade, localizada na latitude Sul entre $23^{\circ} 08^{\prime} 47^{\prime \prime}$ " e $23^{\circ} 55^{\prime} 46^{\prime \prime}$

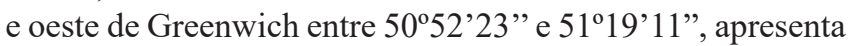
área territorial de $1.650,809 \mathrm{Km}^{2}$, sendo $264,641 \mathrm{~km}^{2}$ de área urbana, onde estão residentes 493.520/506.701 (97,39\%) habitantes, sendo considerada a segunda maior cidade do estado do Paraná, sul do Brasil. O Índice de Desenvolvimento Humano (IDH) ranqueia o município na sexta maior colocação do Estado do Paraná (IBGE, 2015).

A pesquisa de enteroparasitas, foi realizada em amostras de fezes de voluntários, residentes na área urbana do município, com idade superior a 18 anos, após consentimento livre e esclarecido (CEP/UEL1.025.861/2014).

Para a coleta do material foi entregue a cada participante um frasco tampado, limpo e identificado. No dia agendado, as amostras de fezes frescas foram recolhidas, mantidas sob refrigeração e encaminhadas ao Laboratório de Parasitologia da Universidade Estadual de Londrina, para realização dos exames.

Para cada amostra foram utilizadas técnicas de Sedimentação espontânea (HOFFMANN et al., 1934), para detecção de ovos e larvas de helmintos, e de Centrífugo-flutuação (FAUST et al., 1939) para pesquisa de cistos de protozoários e de ovos leves de helmintos e a flutuação em solução saturada de cloreto de sódio (WILLIS, 1921), para a pesquisa de ovos de ancilostomídeos.

Dados epidemiológicos foram obtidos por meio de um questionário aplicado a cada participante com perguntas relacionadas ao ambiente e características pessoais como gênero, idade, renda familiar, hábitos de higiene pessoal, dos alimentos e do ambiente, ocorrência de diarreia nos últimos sete dias, abastecimento e armazenamento de água na residência, sistema de esgoto, descarte de lixo, presença de roedores, de pernilongos e carrapatos. 


\section{Resultados}

Participaram do estudo 187 indivíduos residentes na área urbana de Londrina. Foram detectados ovos ou cistos de parasitas intestinais em 8/187 (4,27\%) amostras de fezes, sendo caracterizados 7/8 (87,50\%) como Giardia spp. e 1/8 $(12,5 \%)$ como Enterobius vermicularis. Não foi detectado poliparasitismo nas amostras.

As amostras positivas eram provenientes de seis voluntários do sexo feminino e dois do sexo masculino, com idades entre 18 e 88 anos. Quanto à renda familiar mensal, seis indivíduos estavam inseridos em famílias com ganhos de um a três salários mínimos e dois relataram participar de famílias com ganho acima de três salários mínimos.

Foi relatado por sete voluntários a lavagem das mãos todas as vezes antes da alimentação e após utilizarem o banheiro, enquanto um participante relatou esta prática, porém não todas as vezes. A variável: consumo apenas de água mineral, assim como a ocorrência de diarreia foi relatado apenas por um indivíduo.

Todos os indivíduos relataram abastecimento de água e fornecimento de rede de esgoto em suas residências.

\section{Discussão}

A ocorrência de parasitas intestinais em humanos é relatada desde 1681, quando o pesquisador Antonie Van Leewenhoek relatou encontrar "animalículos" ao analisar suas fezes em microscopia ótica.

A eliminação fecal destes microrganismos pode contaminar o ambiente, solo, água e consequentemente os alimentos, que, quando ingeridos sem tratamento ou higienização, promovem a reinfecção ou a infecção de novos indivíduos (REY, 2002).

As condições climáticas, de infraestrutura, social, econômica e cultural influenciam na frequência de humanos com parasitas de ciclo fecal-oral (SILVA et al., 2016). Nesta análise foi verificado que quatro residências estavam em um mesmo bairro da zona oeste do município, com 322 a 1100 metros de distância entre elas, enquanto as outras quatro, localizadas em diferentes regiões da cidade, se distanciavam em pelo menos 3746 metros entre elas (Figura 01). Sugere-se a existência de variáveis de risco de origem ambiental influenciando a ocorrência de parasitoses intestinais na região.

Figura 1: Distribuição geográfica das residências com humanos com enteroparasitas. A. Região metropolitana de Londrina, PR. B. Distribuição dos oito casos na área urbana do município. C. reprodução ampliada do quadro B. D. Zona urbana com maior concentração dos casos.

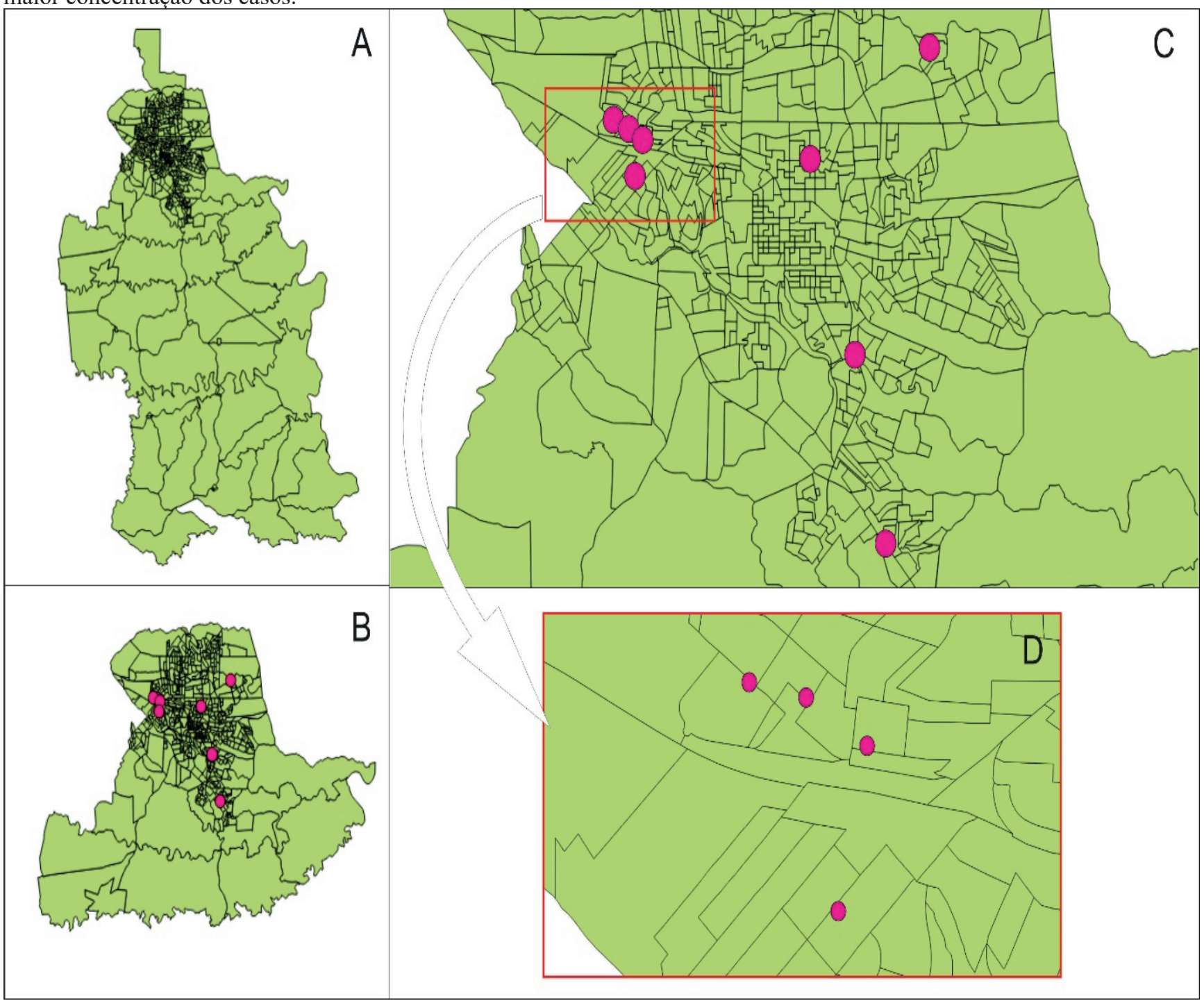


A ocorrência de parasitoses intestinais na cidade de Londrina foi de 8/187 (4,27\%). Comparando este estudo a outros realizados no Estado do Paraná, os resultados foram inferiores aos relatados em Maria Helena em 69/431(16,00\%) amostras. A diferença encontrada pode estar influenciada pela amostragem escolhida, pois, no presente estudo os indivíduos foram selecionados aleatoriamente, enquanto o outro utilizou dados laboratoriais, que geralmente analisam amostras de indivíduos sintomáticos, e que tem alta probabilidade de ocorrência de infecção parasitária.

Outro estudo paranaense relatou prevalência geral em 108/775 (13,94\%) indivíduos selecionados aleatoriamente no município de Marialva, sendo 3,26 vezes superior a encontrada no presente estudo. A detecção de enteroparasitas em humanos pode ser considerada como indicador das condições econômico-sociais de uma população, o que explica a baixa parasitemia detectada é explicada pelos altos índices econômico-sociais de Londrina, que possui o sexto maior IDH do Estado (PNUD, 2010). Outro fator que pode ter influenciado é a presença de agua tratada e de galerias de esgoto em 100\% das casas investigadas. Ludwig et al. (1999) detectaram um declínio na prevalência de parasitoses intestinais em humanos residentes em Assis, SP após melhorias na infra-estrutura sanitária do município. Entretanto, foi observado que em Londrina, o protozoário flagelado Giardia spp. acometeu 7/187 (3,74\%) participantes, e em Marialva foi descrito em 6/775 (0,75\%) das amostras. Esta análise demonstrou que, apesar do menor parasitismo intestinal, a detecção de Giardia spp. superou em 4,86 vezes a relatada em Marialva, evidenciando a importância deste protozoário nas infecções intestinais de Londrina.

A maior detecção de Giardia spp. entre mulheres parece ser causal e não estar associada ao risco de infecção, como verificado por outros autores (ARANI et al., 2008, MINETTI et al., 2015).

Quanto a idade e apresentação clínica dos infectados, em recente estudo sobre a diversidade molecular da Giardia duodenalis, identificou diferença estatística entre os assemblages A ou B e a faixa etária acometida (MINETTI et al., 2015), porém, as informações que associam os genótipos de Giardia spp. com fatores de risco ainda estão sob investigação por diversos pesquisadores ao redor do mundo (RYAN; CACCIO, 2013).

As variáveis associadas ao risco de infecção humana por Giardia spp. derivam do modo de transmissão do parasita, que pode ser diretamente pela via oral-fecal ou indireta, por meio do consumo de água ou alimentos contaminados.

As características observadas nos indivíduos deste estudo foram detectadas como promotoras de giardíase nas comunidades em outros estudos, como: baixo rendimento mensal (VALVERDE et al., 2011), falta de higienização das mãos antes da alimentação (Puebla et al., 2015) e ingestão de água não tratada (SILVA et al., 2016).

O relato de sintomas compatíveis com giardíase foi verificado em apenas uma pessoa, com diarreia na semana anterior à coleta. É sabido que a doença pode manifestar quadro clínico variável, desde a presença de diarreia crônica ou aguda, desidratação, dor abdominal, náusea, vomito e perda de peso e má absorção intestinal até quadros assintomáticos (CACCIO; RYAN, 2008).

A ausência de sintomas em indivíduos infectados e com eliminação dos ovos de parasitas é importante fator ambiental que aumenta o potencial de disseminação dessa verminose, pois o indivíduo passa a atuar como fonte de infecção (MONTRESOR et al., 2012; COLLI et al., 2013).

Outra importante fonte de infecção são os animais domésticos e silvestres, que, ao se infectarem, podem eliminar os cistos de Giardia spp. através das fezes e contaminar o ambiente, de forma semelhante ao humano. A participação de animais no ciclo parasitário faz a doença adquirir caráter zoonótico (FENG; XIAO, 2011).

Colli et al. (2013) e Valverde et al. (2011), verificaram que ao compartilhar o espaço domiciliar com animais infectados, respectivamente, duplica e triplica a chance de infecção por humanos. Puebla et al. (2015) detectaram a presença do cão na casa como fator de risco para giardíase nos humanos, e Johnston et al. (2010), apontaram que a infecção por Giardia spp. pode ser observada em humanos e em seus animais de estimação quando residem próximos à áreas com primatas infectados.

Nos últimos anos, pequenos mamíferos silvestres estão se tornando popular como animais de estimação, despertando para uma importante questão sobre a possibilidade desses animais atuarem como fonte de infecções parasitárias zoonóticas no ambiente domiciliar (MONIS et al., 2009). Para identificar a possibilidade do risco de infecção zoonótica, pesquisadores como Soares et al. (2008) e Pantchev et al. (2014) investigaram a presença de parasitas intestinais a partir de amostras de fezes destes animais e detectaram cistos de Giardia spp. em ouriço e em coelhos, chinchilas, porquinhos da índia e furões.

Para reduzir os riscos de infecção diante dessa nova interação entre humanos e animais de estimação, é fundamental que a saúde da família seja monitorada de forma única, com interação entre profissionais da área humana e animal (ASOKAN et al., 2012).

A inserção do médico veterinário junto ao NASF (Núcleo de Assistência à Saúde da Família), orientando a população quanto à guarda responsável dos aninais de estimação, com vacinação, vermifugação, controle populacional, cuidados higiênico-sanitários, poderia contribuir para a identificação dos fatores de riscos para infecção e prevenção de zoonoses.

A detecção de Enterobius vermiculares em 1/187 $(0,53 \%)$ amostra de fezes pode ser interpretada como indicador de contaminação e sugere a existência de uma fonte de infecção na área urbana de Londrina, que permite a ocorrência de um ciclo parasitário oral-fecal.

Ao extrapolar os resultados $(4,27 \%)$ para a população residente em área urbana do município (484.456), é esperado que 20.686 pessoas estejam infectadas por algum enteroparasita, contaminando o ambiente e mantendo a infecção na área estudada e sem diagnóstico de doença.

É sugerida a inserção do médico veterinário nas ações de Saúde Única nesta comunidade para identificar os fatores de risco associados a zoonoses e reduzir a incidência de infecções em humanos.

\section{Conclusão}

Na população urbana estudada ocorrem infecções assintomáticas por enteroparasitas com predomínio de pa- 
rasitismo pelo protozoário flagelado Giardia spp. Ações em Saúde Única são sugeridas para incrementar a saúde da comunidade.

\section{Referências}

ARANI, A. S. et al. Prevalence of intestinal parasites in a population in south of Tehran, Iran. Revista Instituto de Medicina Tropical de São Paulo, v. 50, p. 145-149, 2008.

ASOKAN, G. V. et al. One health: perspectives on ethical issues and evidence from animal experiments. Eastern Mediterranean Health Journal, v. 18, p. 1170-1173, 2012

BORGES, W. F. et al. Parasitos intestinais: elevada prevalência de Giardia lamblia em pacientes atendidos pelo serviço público de saúde da região sudeste de Goiás, Brasil. Revista de Patologia Tropical, v. 40, n. 2, p. 149-157, 2011.

BRASIL. (2012). Ministério da Saúde. Plano integrado de ações estratégicas de eliminação da hanseníase, filariose, esquistossomose e oncocercose como problema de saúde pública, tracoma como causa de cegueira e controle das geohelmintíases. Plano de Ação 201 1-2015. 2012.

COLLI, C. M. et al. Prevalence and risk factors for intestinal parasites in food handlers, southern Brazil. International Journal of Environmental Health Research, v. 24, n. 5, p. 450-458, 2014.

FAUST, E. C. et al. Comparative efficiency of various techniques for the diagnosis of protozoa and helminth in feces. Journal of Parasitology, v. 25, p. 241-261, 1939.

GOMES, K. B. et al. Giardia duodenalis: genotypic comparison between a human and a canine isolates. Revista da Sociedade Brasileira de Medicina Tropical, v. 44, n. 4, p. 508-510, 2011.

HOFFMANN, W. A. et al. The sedimentation concentration method in Schistosomiasis mansoni. Puerto Rico. Journal of Public Health \& Tropical Medicine. v. 9, p. 283-298, 1934.

IBGE: Instituto Brasileiro de Geografia e Estatistica. Disponível em: http:/www.ibge.gov.br/cidadesat/ topwindow.htm?1> Acesso: 24 de agosto 2016.2015.

LOPES, F. M. R. et al. Occurrence of enteroparasitosis in schoolchildren of the municipal district ofJataizinho, State of Paraná, Brazil. Acta Scientiarum Health Science, Maringá, v. 28, n. 2, p. 107-111, 2006.

LUDWIG, K. M. et al. Correlação entre condições de saneamento básico e parasitoses intestinais na população de Assis, Estado de São Paulo. Revista da Sociedade Brasileira de Medicina Tropical, v. 32, n. 5, p. 547-555, 1999.

MINETTI, C. et al. Determination of Giardia duodenalis assemblages and multi-locus genotypes in patients with sporadic giardiasis from England. Parasites \& Vectors v. 8, p. 444.2015 .

MONIS, P. T. et al. Variation in Giardia: towards a taxonomic revision of the genus. Trends in Parasitology, v. 25, n. 2, p. 93-100, 2009.

MONTRESOR, A. et al. Helminth control in school-age children: a guide for managers of control programmes. Geneva, World Health Organization, 2002.

NEVES, D. P. et al. Parasitologia humana. São Paulo, Atheneu. p.494, 2005.

PANTCHEV, N. et al. Occurrence and molecular typing of Giardia isolates in pet rabbits, chinchillas, guinea pigs and ferrets collected in Europe during 2006-2012. Veterinary Record. v. 175, n. 1, p.18-22, 2014.

PNUD. Programa das Nações Unidas para o Desenvolvimento- PNUD. Atlas do desenvolvimento Humano no Brasil 2013 (Dados de 2010). Disponível em: https://nacoesunidas.org/pnud-lanca-relatorio-dedesenvolvimento-humano-2015-com-foco-nos-desafiosdo-novo-mundo-do-trabalho/. Acesso em: 15 de agosto de 2016.

PRADO, M. S. et al. Prevalência e intensidade da infecção por parasitas intestinais em crianças na idade escolar na Cidade de Salvador - Estado da Bahia, Brasil. Revista da Sociedade Brasileira de Medicina Tropical, v. 34, p. 99101, 2001.

PUEBLA, L. J. et al. Molecular characterization and risk factors of Giardia duodenalis among school children from La Habana, Cuba. Journal of Parasitology Research, p.18,2015

REY, L. Bases da Parasitologia Médica. Rio de Janeiro: Guanabara Koogan, 380 p. 2002.

RYAN, U.; CACCIÒ, S. M. Molecular epidemiology of giardiasis. Molecular Biochemical Parasitology, v. 160, p. 75-80, 2008.

RYAN, U.; CACCIÒ, S. M. Zoonotic potential of Giardia. International Journal for Parasitology, v. 43, n. 12-13, p. 943-956, 2013.

SAVIOLI, L. et al. Giardia and Cryptosporidium join the 'Neglected Diseases Initiative. Trends in Parasitology, v.22, p.203-208, 2006.

SILVA, J. B. et al. Spatial distribution and enteroparasite contamination in peridomiciliar soil and water in the Apucaraninha Indigenous Land, southern Brazil.

Environmental Monitoring and Assessment, v. 188, p. 217, 2016.

SOARES, J. F. et al. Parasitismo por Giardia sp. e 
Cryptosporidium sp. em Coendouvillosus. Ciência Rural, v. 38, n. 2 , p. $548-550,2008$.

WHO. World Health Organization. http://www.who. int/mediacentre/factsheets/fs366/en/. Accesso em: 30 de novembro de 2015 .

WHO. World Health Organization. Soil-transmitted helminth infections, 2016.

WHO. World Health Organization. The world Health Report, 1996.

WILLIS, H.H. A simple levitation method for the detection of hookworm ova. Medical Journal of Australia, v. 8, p. 375-376, 1921.

Recebido em: 05.09.2016

Aceito em: 27.12.2016 